\title{
SNPs within the GH signalling pathway are associated with the fast, but not the long term, IGF-I response to GH replacement therapy in $\mathrm{GH}$ deficient adults
}

\author{
Camilla AM Glad 1, 2, Edna J L Barbosa 1, 3, Helena Filipsson Nyström 1, Lena MS Carlsson 2, Staffan Nilsson ${ }^{4}$, \\ Anna G Nilsson 1, Per-Arne Svensson ${ }^{2}$ and Gudmundur J ohannsson ${ }^{1}$

\begin{abstract}
${ }^{1}$ Department of Endocrinology, the Sahlgrenska Academy, University of Gothenburg, Gothenburg, Sweden. 2 Department of Molecular and Clinical Medicine, the Sahlgrenska Academy, University of Gothenburg, Gothenburg, Sweden. ${ }^{3}$ Serviço de Endocrinologia e Metabologia do Hospital de Clínicas da Universidade Federal do Paraná, Curitiba, Brazil. ${ }^{4}$ Department of Mathematical Statistics, Chalmers University of Technology, Gothenburg, Sweden.
\end{abstract}

\section{BACKGROUND}

Growth hormone (GH) deficiency (GHD) in adults is associated with low serum levels of insulin-like growth factor I (IGF-I) and a deteriorated cardio-metabolic profile. GH replacement therapy (GHRT) increases serum IGF-I, an important mediator of the treatment response and safety marker of dose titration. The interindividual variation in treatment response is large and most likely influenced by genetic factors.

\section{AIM}

To test the hypothesis that single-nucleotide polymorphisms (SNPs) in genes within the GH signalling pathway impact on the fast (early) and/or long term IGF-I response to GHRT.

\section{SUBJECTS AND METHODS \\ The GH2 study}

This is an ongoing, longitudinal, prospective study of adult patients with hypopituitarism and severe GHD ( $n=313$; see Table 1A for baseline patient characteristics.) Diagnosis of GHD was performed using standard consensus criteria (Ho KK, 2007). GH dose was individually titrated to attain sexand age adjusted normal serum IGF-I levels. The study was approved by the Ethics Committee at the University of Gothenburg, Sweden, and performed according to the Declaration of Helsinki.

\section{Response definition}

Response to GHRT was defined as the percentage of change in serum IGF-I concentrations.

- Early response at 1 week (change from baseline).

- Long-term response at 6 and 12 months (change from 1 week).

\section{Genotyping}

Using a candidate gene approach, six SNPs (Table 1B) were chosen and subsequently analyzed using either TaqMan or Sequenom SNP genotyping. The GHR exon 3 deleted/fulllength polymorphism was analyzed using tagSNP rs6873545, as previously validated (Glad CA et al, 2010).

\section{Statistics}

Genotype data was analyzed in an additive model. Multiple regression analyses adjusting for sex, age and $\mathrm{GH}$ dose were performed.

\section{CONCLUSIONS}

- GHR and PIK3CB SNPs rs6873545 and rs361072 were associated with the early response to GHRT.

- When dissecting the long-term response into one that depends on the early response, no SNPs were associated with the 6 months and 1 year response to GHRT.

- The effect of genetics on response to GHRT is most likely best captured in the early response.

\section{RESULTS}

- Average genotyping success rate was 98.8\%. Minor allele frequencies (MAFs) and Hardy-Weinberg Equilibrium (HWE) $\mathrm{X}^{2}$ test $p$-values are presented in Table 1B.

- One year of $\mathrm{GH}$ replacement therapy increased serum concentrations of IGF-I (Table 1C; $p<0.001$ ).

- GHR and PIK3CB SNPs rs6873545 and rs361072 were significantly associated with the early IGF-I response to GHRT (Table 1D; $p=0.016$ and $p=0.025$, respectively).

- No SNPs were associated with the long-term response to GHRT, when the 1 week serum IGF-I levels were used as reference (Table 1D).

TABLE 1. Clinical and genetic background information and results.

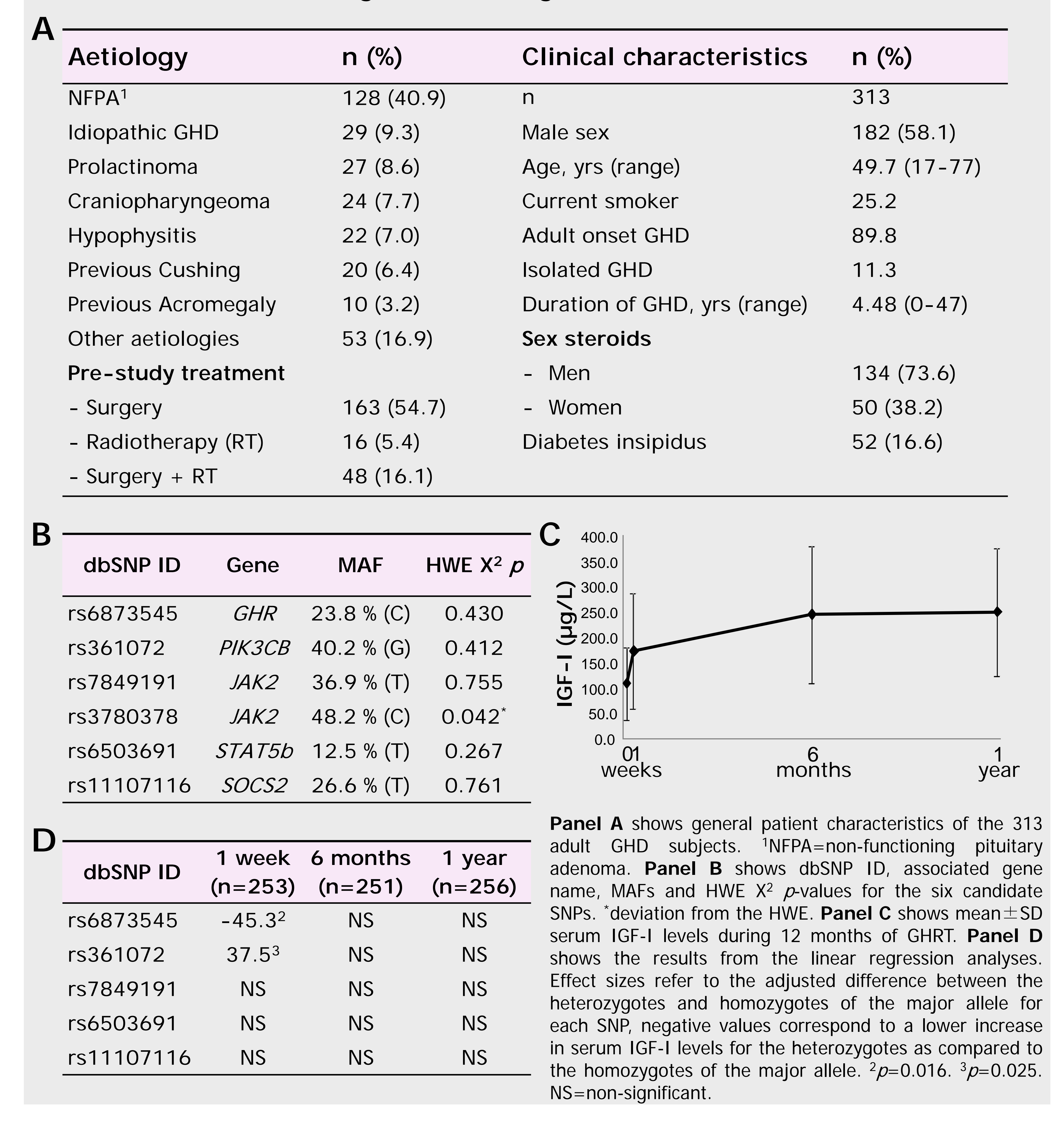

